protrusion and as to the state of development or mal-development of the other structures of the eyeball. The protuberant portion of retina consists of varying amounts from simply a knuckle of tissue to quite two-thirds of that membrane. In some cases it forms a solid mass surrounded and intermixed with bands of fibrous tissue. In others folds. of retina have become distended into one or more fluid-containing cystic protrusions which are bounded externally by a fibrous-tissue coat and have a lobulated outline.

The degree of development of the protuberant retina is also very different in different cases. When folds of it have become distended in the form of cystic protrusions it may be so atrophied as merely to be represented by a few groups of nuclear bodies and a little retiform tissue. On the other hand, it may remain so well developed that some of its different layers are easily distinguished. In the latter cases it is found that sometimes the inner surface of the retina is turned towards the interior of the cyst, and at other times just the reverse, the inner surface of the retina being directed towards its fibrous outer wall. The region of the globe in which the protrusion is situated is nearly always its lower and posterior part, sometimes a little further forwards than others. In one case, besides the gap in the sclerotic there was a considerable deficiency in the outer sieath of the optic nerve.

Speaking generally, the larger the amount of the retinal protrusion the smaller and more imperfectly developed is the eyeball. When the protrasion is not very extensive the eyeball may present little external evidence of malformation beyond its defect in size. The lens and vitreous may also appear quite normal. On the other hand, there are numerous congenital defects which may be met with in association with it, such as vascularity of the cornea, formation of cartilage in the sclerotic, coloboma of iris, persistent pupillary membrane, displacement of ciliary processes, cataract, persistence of blood-vessels in the vitreous and formation of fibrous tissue in it, detachments of the retina elsewhere than at the protrusion, and hyaline nodules in the choroid.

In considering the causation of this congenital malformation I would first like to point out that in none of the seven specimens which I have examined is there anything which would lead me to suppose that the changes shown were inflammatory in origin. In one case there were some coliections of round cells found in the cyst wall which might have been inflammatory; if so they were of recent production, and as the eye was not removed until the patient was 18 years old they cannot be considered as having had anything to do with the causation of the congenital defect. I lay stress on this point because Deutschmann in 1881, from his investigation of a case of bilateral coloboma of the iris and choroid in a rabbit, advanced the theory, which has been widely accepted and quoted, that so-called arrests of development were only changed developments of the eye the result of intra-uterine inflammation. Hess brought forward several cases which, like those which 1 have examined, go to show that inflammation has very much less to do with the production of congenital malformations than has generally been supposed. The difference in the position of the retina in these cases suggests that they are not all produced in precisely the same way. When the normal outer surface of the retina is external in the cyst it is possible, as Alt has suggested, that part of the lower wall of the globe, where there was a defect in the choroid and partial defect in the sclerotic, may have been stretched before the intra-ocular pressure and the lining retina pushed into the depression formed. Where, however, the normal outer surface of the retina is towards the interior of a cyst and where the amount of retina which protrudes is very large, such an explanation cannot be accepted, and Kundrat's view, that the cystic formations are due to the projection during development of a portion of the retinal tissue through the fotal cleft into the subjacent mesoblastic tissue, would seem more applicable. The retina in the human foetal eye is normally thrown into folds and it is not difficult to understand how, as the result of some imperfect development of the vitreous humour or delayed closure of the cleft, one or more of these folds of the inner layer of the secondary optic vesicle might become extruded through the foetal ocular cleft into the surrounding mesoblast, and subsequently become expanded into one or more fluid-containing cysts.

\section{A CASE OF INTERSTITIAL FIBROID TUMOUR SLOUGHING "EN MASSE" AND SOME OTHER CASES OF FIBROID TUMOURS OF THE UTERUS. ${ }^{1}$}

BY ARTHUR H. N. LEWERS, M.D. LOND., OBSTETRIC PHYSICIAN TO THE LONDON HOSPITAI.

AMONG the following nine cases of fibroid tumour of the uterus Case 7 is especially interesting on account of its rarity. It was a case where a large interstitial fibroid tumour situated in the posterior wall of the body of the uterus suddenly sloughed and the sloughing mass protruded, not through the os uteri, but through an opening spontaneously formed in the posterior lip of the cervix. As no operation was done the case may, I think, fairly be classed as one in which a fibroid tumour of the uterus was the direct canse of the patient's death. Such a thing is certainly a rare event, though perhaps not quite so rare as is sometimes supposed. The other cases are interesting cliefly as typical examples of current methods of treatment.

Case 1 was one of uterine fibroids complicated by suppurating ovarian cyst; both sets of uterine appendages were removed and the fibroid has diminished very much in consequence. Cases 2,3 , and 9 are instances of abdominal hysterectomy with intra-peritoneal treatment of the stump. In Case 3 a point of interest was that the fibroid had under. gone cystic change and that one cavity so produced contained puriform fluid. Case 4 was one of pregnancy complicated with large subperitoneal fibroid, in which expectant treatment was adopted with a good result as regards the labour and in which at present symptoms are absent. Cases 5 and 6 illustrate the removal by morcellement of submucous fibroids from the cavity of the body of the uterus after dilatation of the cervix; and Case 8 is an example of myomectomy for large subperitoneal fibroid with intra-peritoneal treatment of the stump. In this case the diagnosis between fibroid and ovarian tumour was doubtful till the abdomen had been opened.

CASE 1. Suppurating ovarian tumour (left side) and fibroid tumours of the uterus; ovariotomy (left side) and removal of uterine appendages on the right side; marked diminution in size of the fibruid after operation with complete relief of symptoms.-The patient, a single woman, aged 37 years, was admitted under my care into the London Hospital on Jan. 11th, 1897. She was sent to me by Dr. Deane of Leytonstone. About two years previously she noticed that the lower abdomen was bigger than it ought to be, but she never felt any particular lump there. For the previous two years the quantity lost at the menstrual periods had been excessive and for the previous month there had been a constant sanious discharge. A month previously she noticed swelling of both legs, principally the left, also aching in the legs. Both conditions were somewhat relieved by lying down. During the same time (one month) there had been pain in the lower abdomen, which was chiefly referred to the left iliac region. The catamenia began when she was fourteen years of age and had recurred regularly every four weeks till the beginning of the present illness. The quantity lost was moderate and there was very little pain at the periods; what little pain she had occurred on the first day of the period and was relieved as the flow became established. Her general health bad been good till the present illness began.

On admission the patient was found to be thin, sallow, looking, and anæmic. On abdominal examination a hardirregular swelling was felt in the lower abdomen rising out of the pelvis and reaching half way to the umbilicus. It extended outwards to within two inches of the left anterior superior iliac spine. On vaginal examination the tumour in the hypogastric region was found to be the body of the uterus, considerably enlarged, and a good deal less moveable than it should be. There was a softer swelling in the left lateral fornix such as might be produced by a dilated Fallopian tube or a small ovarian cyst. The sound passed three inches and five-eighths of an inch with some difficulty. The case

A paper read before the Harveian Society of London on Nor. 16th, 
appeared to me to be one of uterine fibroids probably complicated by a small ovarian cyst on the left side. The patient was kept in bed for nearly three weeks prior to operation with tne view of improving her general condition, and during this time the swelling in the legs became much less marked.

Operation was performed on Feb. 5th, 1897. The abdomen was opened in the usual way. The central tumour, as was expected, was then seen to be the uterus enlarged by fibroids, and there was an ovarian cyst on the left side of the size of a cocoanut, which was intimately adherent to the sigmoid flexure and in the pelvis. During the separation of the cyst it burst and pus escaped. A satisfactory pedicle was obtained and the ovarian cyst was removed in the usual manner. The uterine appendages on the right side were practically normal, but I thought it well to remove them with the object of influencing the uterine fibroids. As some pus had escaped during the removal of the cyst the peritoneum was washed out with warm normal saline solution and a drain of iodoform gauze was left in the pelvis; the rest of the wound was closed as usual. Examination of the parts removed from the left side showed that the left Fallopian tube was greatly thickened so as to be equal in diameter to the ball of the thumb. No trace of its fimbriated extremity could be seen, the outer end of the tube being firmly attached to the suppurating ovarian cyst, but not communicating with it. The patient made an uneventful recovery and left the hospital on March 6th, 1897.

I have seen the patient on several occasions since and she has remained quite well. There was a slight show about June, 1897; then nothing was seen for nine months, then she had three periods lasting ten days, six days, and four days respectively. When I saw her in Feburary, 1899, there had been nothing seen since March, 1898. The case illus trates that the establishment of the menopause after removal of the uterine appendages may be gradual and he spread over an interval of 18 months or two years. The tumour has diminished in size very much. Only on deep pressure can anything be felt of it at all on abdominal examination alone.

C.ASE 2. Abdominal hysterectomy with intra-peritoneal treatment of the stump; uterus enlarged by multiple fibroids forming a large abdominal tumour extending to the epigastric region; good recovery.-A married woman, aged 43 years, was admitted under my care into the London Hospital on Nov. 11th, 1898. She was sent to me by Dr. Fennell of Tulse-hill. The patient stated that she had been married five years but had not been pregnant. Her husband was 69 years old. The catamenia began when she was about 15 years of age and had recurred quite regularly till three months previously. She always had lost a rather large quantity at the periods and as a rule they lasted from seven to nine days. Till ten years previously the periods were accompanied by biliousness and sickness. During the three months preceding admission the periods had occurred everv fortnight and had lasted a fortnight, so that there had been an almost continuous loss of blood. The last period occurred a month previously and lasted three weeks. There was then a week without any loss and then it began again. There had been no dysmenorrhoea. She first noticed a swelling in the abdomen 12 months previously but thought it was due to flatulence. Six weeks previously she consulted her own medical man as the swelling had rapidly increased. She had noticed that the swelling became larger just before the period and decreased slightly when the flow began. She had lost flesh of late.

On examination the abdomen was seen to be prominent especially on the right side from the epigastrium downwards. The surface of the prominence was slightly irregular. The highest point of the tumour was $13 \frac{1}{2}$ inches from the pubes measured along the convexity. The girth at the umbilicus was 34 inches; three inches lower down it was 35 inches. The umbilicus was level with the skin. On palpation a hard irregular tumour was felt rising from the pelvis and reaching the level mentioned above in the epigastric region. One portion of the tumour which measured three inches by two inches just to the right of the umbilicus could be moved rather freely on the deeper seated portion of the mass. The whole tumour could be moved a little from side to side and also about one and a half inches upwards. A well-marked uterine sounle could be heard over various parts of the tumour. On vaginal examination there was seen a good deal of recent blood about the parts. The hymen was tight and the vacina was greatly distended. The vaginal portion of the cervix was so much drawn up that the os uteri could only just be touched even when the tumour in the abdomen was pushed towards the pelris. Under the circumstances I did not try to pass the sound a it seemed impossible to do so without giving the patient an anæsthetic and the diagnosis was obvious without it.

Operation was performed on Nov. 13th, 1898. The abdomen was opened by a long median incision extending from the middle of the epigastric region to within one and a balf inches of the pubes. The tumour was then brought outside and the upper portion of the incision as far as the umbilicus was then closed with silk sutures. The upper portion of the right broad ligament was then ligatured in two places and the part between the ligatures was divided. The outer of these ligatures was so placed that the right ovary was not removed. The left broad ligament was treated in a similar way and divided, but on this side the ligatures were so placed that the left ovary remained attached to the tumour and was subsequently removed with it. Anterior and posterior flaps of peritoneum were then made, the flups being stripped to what was judged to be the level of the internal os uteri. The uterine arteries, which could be well seen, were then tied at this level and the body of the uterus with the fibroids was cut away. The stump consisted of the cervix; its cut edges were approximated with stout silk sutures and the peritoneal flaps were then stitched over the stump by numerous interrupted Lembert's sutures. A Keith's drainage tube was inserted and the rest of the abdominal wound was closed. The patient had some slight rise of temperature during the first few days after the operation, but at the end of the first week the temperature was normal. She subsequently did quite well and she left the hospital on Dec. 14th, 1898.

The tumour weighed 7 pounds 11 ounces some time after it had been cut into and after what fluid it contained had drained away. It consisted of the uterus greatly enlarged by multiple fibroids. The cavity of the uterus was much elongated, so that the sound would have passed seven or eight inches. The specimen illustrated beautifully every variety of fibroid except submucous fibroids with distinct neck and fibroid polypi ; but peritoneal fibroids, interstitial fibroids, and tumours partly submucous were all represented.

I heard from this patient a year after the operation that she was in very good health and during the past year had been able to nurse her invalid husband, which she certainly could not have done in the state of health existing prior to the operation.

OASE 3. Large abdominal tumour composed of uterus nith large sub-peritoneal fibroids, one of which had undergone cystic change with formation of puriform fluid; abdominal hysterectomy with insra-peritoneal treatment of the stump; good recovery.-A single woman, aged 38 years, was admitted into the London Hospital under my care on Jan. 31st, 1899. She was sent to me by Dr. Surtees Sumner of Guilford-street, Russell-square. The patient first noticed a small lump of the size of a pigeon's egg in the right side of the abdomen five years previously. It then caused no pain or inconvenience and she did not trouble to consult a medical man about it. The lump gradually increased in size and about three years previously she thought it moved to the left side. It had increased more rapidly during the last year and had become very hard. She had also had pain in the region of the tumour just before menstruation. On Dec. 31st, 1898, she was seized with a violent attack of pain in the abdomen; she then sent for her medical adviser and she was in bed about a week. The pain was so severe that she could not stand upright. It was reliered by hot flannels and hot-water bottles. She had a second attack of pain similar to the first, but not so severe, a week before admission. Her general health had been as a rule good. In 1892 she had influenza followed by pneumonia and phlebitis. During the last year she had lost flesh considerably. Menstruation began when the patient was 16 years of age and she had been quite regular every four weeks, losing a normal quantity and not having much pain. She had not menstruated since November, 1898. On admission the abdomen was found to be irregularly prominent, the most prominent part being at the right of, and a little below, the umbilicus. On palpation the greater part of the abdomen was found to be occupied by a hard irregular tumour. It had a fair degree of mobility from side to side and also slight mobility up and down. Nothing was heard orer the tumour. On raginal examination the hymen was found to 
be extremely tight and was torn by examination. The cervix was high up, far back, and consequently difficult to reach. A hard mass was felt in the anterior fornix, apparently continuous with that in the abdomen; it had some degree of mobility. The sound was passed with great difficulty a distance of three inches.

Operation was performed on Feb. 2nd, 1899. The abdomen was opened by the usual median incision, beginning just below the umbilicus to within one and a half inches of the pubes, but before the tumour could be brought outside the wound had to be extended nearly to the ensiform cartilage. When the tumour had been drawn outside the wound the npper part of the incision as far as the umbilicus was at once closed. The remaining steps of the operation were similar to those in Case 2. The left ovary was not removed.

The tumour weighed nine pounds. It consisted principally of two subperitoneal fibroids of unequal size attached to the body of the uterus, which was moderately enlarged. The larger of these was attached to the uterus by a pedicle so thin that it could be rotated through almost a complete circle. The smaller was attached by a thicker, shorter pedicle, but it could be rotated through two-thirds of a circle. The dimensions of the larger fibroid were: length, nine inches ; breadth, six and a half inches; and thickness, five inches. The dimensions of the smaller were six and a half inches by four and a half inches by three inches respectively. There was besides a small subperitoneal fibroid of the size of an egg attached to the uterus by a pedicle allowing considerable rotation. The portion of the uterus (body) removed with the tumours was three inches long, three inches broad, and one and a half inches thick. On section several smaller fibroids were seen in the body of the uterus. On section of the larger subperitoneal fibroid it was seen to have undergone cystic degeneration at one place producing a cavity the cut edge of which measured two and a half inches by three inches. This cavity contained pus. The smaller subperitoneal fibroid showed evidence of commencing cystic changes.

The patient made a good recovery and she left the hospital on March 1st. The scar measured seven and a quarter inches.

I have seen the patient once since she went out. She was then quite well and had then been able to resume her work.

As regards the attacks of severe pain which were noticed prior to the operation it appears probable that they may have been caused by the axial rotation of either of the large subperitoneal fibroids, which, as mentioned above, were very freely moveable on the long axis. The pain might also have been due to a loop of bowel getting nipped between the two large subperitoneal fibroids. The larger of these had undergone cystic change at one place with formation of puriform finid, and, apart from operation, it is practically certain that other serions symptoms must have arisen before long.

CASE 4. Large subperitoneal fbroid tumour complicating pregnancy; expectant treatment; normal labour; subsequent moderate diminution in sive of the fibroid; absence of symptoms at present.-The patient, a married woman, aged 29 years, was admitted into the London Hospital under my care on April 25th, 1299. She was sent to me by Dr. Dent of Woodford. She stated that she was married 12 months previously and that up till then she had mlways enjoyed very good health. The catamenia began when she was 13 years old and she had always been regular every four weeks, the period lasting five days. The last period had occurred in August, 1898. The patient believed herself to be eight months pregnant. About six weeks after the last period she noticed a swelling in the left side of the abdomen; it was not very big but she had to let out her clothes. This swelling gradually increased till January, 1898, since which time it appeared to have remained stationary. About December, 1898, she noticed another swelling in the right side of the lower abdomen (the pregnant uterus), and this had continued to enlarge up to the date of her admission. On Jan. 17th she had a sudden attack of pain in the right side of the abdomen. The pain was very severe and lasted for nine hours. She had more or less pain for a week after this and then remained free from pain for a month. She then had another attack of pain, not quite so severe as the first and not lasting so long. Since then she had had similar attacks about once a month and had been obliged to remain in bed since January-i.e., three months.

I may say that I saw the patient in consultation with her own medical man in January and I diagnosed a large subperitoneal fibroid on the left side complicating pregnancy, and expressed the opinion that there was no reason for interrupting the ordinary course of the pregnancy. On admission the abdomen was found to be irregularly enlarged. There were two prominences divided by a depression; the larger one to the right was the pregnant uterus. The tumour of the left side extended well down into the flank; it met the pregnant uterus in the middle line and extended as low as a finger's breadth from the left anterior superior iliac spine. It was smooth in outline, hard, and slightly tender. There were various areas of resonance between the uterus and the tumour corresponding to the depression between them. As low as the umbilicus the tumours were easily differentiated, but below that level they appeared to merge into one. $\mathrm{On}$ vaginal examination there were the ordinary signs of pregnancy and there was no evidence that the tumour encroached on the true pelvis.

On June 7 th the patient was delivered naturally of a female child. There was no post-partum hæmorrhage. On the 17th the highest part of the tumour to the left seemed to be about two fingers'-breadth below the ribs in the left nipple line. The uterus lay to the right of this swelling but could not be separated from it. The patient went home on June 21st, 1899, with directions to come to the hospital in two or three months. She did so, and on examination then the tumour to the left had become very much smaller, but still formed a swelling that could be felt in the lower abdomen on abdominal palpation alone. It seemed on bimanual examination to be connected with the uterus and to have all the characters of a sub-peritoneal fibroid. As the patient was feeling perfectly well and not complaining of any inconvenience at all I advised her for the present not to have anything done, but to come up to the hospital again if she had any symptoms.

CASE 5. Menorrhagia and metrorrhagia aue to submucous fibroid of the body of the uterus; marked ancemia; dilatation of the cervix and removal of the fibroid by morcellement and enucleation; good recovery.-A married woman, aged 38 years, was admitted into the London Hospital under my care on May 13th, 1898. The patient was sent to me by Dr. O. C. Scott of Twickenham. She had been married four and a half years lout had had no children. There was one miscarriage nearly three years previously, when she was about three months pregnant. This followed a slight accident. The patient had not been quite herself since the miscarriage. Any little orer-exertion caused her, as she said, "to come on unwell." The bleeding was stopped if she was able to rest. She was regular if she could rest up to Christmas, 1897. The first period after this lasted a fortnight; since then there had been irregular hæmorrhage, only going a week or two between the losses. She had lost a great deal of blood since Christmas and had had severe pain in the lower part of the back.

On admission the patient was very markedly anæmic, the mucous membranes being very white. On examination slight bleeding was still found to be going on; the uterus was enlarged, but the os uteri was not patulous. A specially prepared laminaria tent was inserted with all antiseptic precautions and the next day the patient was anæsthetised and the dilatation of the cervix completed with Hegar's dilators up to No. 26. The finger was then passed into the uterus and a submucous fibroid of the size; of an orange was found attached on the posterior wall of the uterus. Although the greater part of the tumour was no doubt projecting into the cavity of the uterus a considerable portion of it was embedded in the uterine wall. The tumour was removed with considerable difficulty by cutting away piece after piece of it with long scissors and enucleating the embedded portion from the wall of the uterus with the finger. The pieces removed were collected and tied up tightly in a layer of gauze in the shape of a ball, thus giving a rery good idea of the size of the tumour within the uterus before the operation. I could not detect the presence of any other fibroid. The patient subsequently did very well; she had no rise of temperature after the operation.

OAsE 6. Menorrhagia and metrorrhagia due to submucous fibroid in the body of the uterus; dilatation of the cervix. remoral of the tumour by morcellement; gnow recovery. A single woman, aged 44 years, was admitted under my care into the London Hospital on July 14th, 1899. The patient was sent to me by Dr. McMullen of Brixton. She stated that she was quite well and that menstruation was perfectly regular till two years before admis sion. She then noticed that the loss of blood at the periods was greater than it had been before. This went on for a year and did not affect her much. During the 
past twelre months not only had the loss at the periods increased but she had had losses of blood between the periods. These losses at first only occurred occasionally, but latterly she had had a constant loss of slight quantities of blood between the periods and also she had had a dull aching pain in the left iliac region. This pain had been worse during menstruation, especially just before the flow became fully established. She had not lost flesh but felt somewhat weaker owing to the constant loss of blood.

On examination the uterus was found to be distinctly enlarged in the normal position and freely moveable. The external os was not patulous; there was a small mucous polypus at the external os of the size of a pea. The cervix was dilated as in the preceding case and a submucous fibroid of the size of a Tangerine orange was removed by enuclea tion and morcellement. The patient made an uninterrupted recovery and left the hospital on August 16th.

CASE 7 Septicemia due to spontaneous sloughing "en masse" of an interstitial fibroid in the posterior nall of the body of the uterus; spontaneous extrusion of a portion of sloughing tumour through an opening in the posterior lip of the cerrix; manual removal of the remainder; continued septic symptoms and death at the end of three :nonths.-A married woman, aged 40 years, was admitted into the London Hospital on July 20th, 1899. The patient was sent to me by Dr. Charles Grant. She had been married $12 \frac{1}{2}$ years and had had one child eight years previously, but had had no miscarriages. The patient had never been really strong, but the present illness dated from Christmas, 1898. The first thing that she noticed wrong was that her menstrual period then lasted three weeks as compared with four or five days, its previous duration. She sent for her medical man who ordered her to stay in bed and gave her some medicine. Between Christmas, 1898, and April, 1899, she had had two similar attacks, each lasting three weeks, and which caused her to feel so ill that she was obliged to stay in bed. In April, May, and June the periods lasted longer than they should have done, but the loss was not so great as during the attacks above mentioned I saw the patient for the first time on May 30th and found the uterus enlarged by fibroids so as to form an abdominal tumour reaching nearly to the umbilicus. The os was a little patulous, but the vaginal portion of the cervix was not at that time at all invaded by any of the fibroid tumours. The patient was advised to take ergotin pills and a mixture containing tincture of hydrastis canadensis About the third week in July, some six weeks after I had first seen her, her medical attendant wrote to me to say that the patient had an extremely offensive discharge and that on examination he found the upper part of the vagina occupied by a brittle offensive mass so that he was afraid she nad cancer. The patient was admitted to the hospital a few days afterwards-i.e., on July 20th.

On admission she was found to have a horribly offensive discharge and the temperature was $101^{\circ} \mathrm{F}$. She was very anæmic and looked very ill. On examination the uterus was felt reaching to within a finger's breadth of the umbilicus. The upper half of the vagina was occupied by a large mass generally convex downwards, very irregular in places, and very friable, so that it could be easily broken down with the finger. Under an anæsthetic a better examination was made. The external os was a slit-like opening in front of the offensive mass. The os readily admitted the finger. The anterior lip of the cervix was very thin with a sharp edge, but the posterior lip could not be recognised as such. The sloughing fibroid was not coming through the os at all, but had evidently gradually made its way through the whole of the posterior wall of the cervix, and had then burst through an opening which had formed on the posterior lip of the cervix itself. The sound passed five and a half inches. The patient was in a profoundly septic condition, as may easily be understood. She seemed to me to be in much the same condition as a patient after confinement where the placenta has not been removed and has decomposed, except that in the present instance the decomposing mass was not in the cavity of the uterus at all, but embedded in the posterior uterine wall, except the lowest portion of it, which had burrowed through the posterior lip of the cervix, producing the fungating mass occupying the upper part of the vagina. All I did in the case was to remove handfuls of the sloughing mass with the fingers, the pieces removed weighing two pounds and eight ounces; the manipulation was to some extent like that of remoring a putrid placenta. The "bed" from which the mass was remored was mopped out thoroughly with tincture of iodine asd washed thoroughly with iodine water. The carity of the uterus was also washed out with iodine water. The uterus, which before removal of the sloughing mass had reached to within a finger's breadth of the umbilicus, contracted down like a uterus after a five months miscarriage so as to lie completely in the pelvis. Subsequently for a long time vaginal douches of iodine water were given every four hours and the uterus was also washed out once or twice a day for a considerable time. The patient was, however, in a profoundly septic condition on admission, and although she lived for some three months after the expulsion of the mass, fever persisted more or less and she finally died worn out by it.

The only alternative in the way of dealing with the case when the sloughing fibroid was found projecting through the posterior lip of the cervix as described would have been to let it alone to come away by itself. What the chance of recovery would have been with a putrid mass of tissue weighing two pounds eight ounces embedded for the most part in the posterior wall of the body of the uterus may be easily imagined. Although the patient finally died three months afterwards I think that removing the mass as soon as possible gave her the only chance of recovery. The case appears to me to be an extremely interesting one for many reasons. The spontaneons sloughing and extrusion en masse of a large intestinal fibroid such as that described is a rare event; I have not myself in nearly 15 years' experience at the London Hospital met with a similar case. Again, the direction in which the extrusion took place-i.e., through the substance of the posterior lip of the cervix-is a remarkable thing. The more natural direction wonld seem to be through the mucous membrane of the body of the uterus. Whether the course of treatment-of ergotin and hydrastis-had anything to do with the sloughing of the mass I cannot say. These drugs are very commonly prescribed in cases of fibroids and for long periods of months and even years without producing any such effect. Again, I feel little doubt that if abdominal hysterectomy had been performed when I first saw the case the patient would have been alive now. The occurrence of such a case illustrates at all events that what may be called the expectant treatment of large fibroids has certain definite risks and that at present it is impossible to say as regards any particular case whether such an accident as sloughing en masse is likely to occur or not.

CASE 8. Large subperitoneal fibroid (five pounds and six ounces) with a thick pedicle; adthesions to several inches of intestine: removal of the tumour and uterine appendages on both sides (hydrosalpinx left side); good recovery.-The patient, a married woman, aged 50 years. was admitted into the London Hospital on July 31st, 1899. She was sent to me by Dr. Spencer of Stoke Newington. She had been married 24 years and had had three children, the last 17 years previously. She had had no miscarriages. Menstruation had always been regular and normal up to the time of admission to the hospital. The patient stated that she hac noticed that the abdomen had been getting larger for the previous two years and much larger during the previous six months. She had also had pain in the right side of the abdomen for some time. Her general health had usually been good.

On admission a large, somewhat rounded tumour was felt occupying the greater part of the abdomen and extending to within three and a half inches of the costal angle. The tumour was hard and nearly symmetrically situated as regards the middle line, extending outwards on each side to within a short distance of each anterior superior iliac spine. The surface of the tumour was slightly irregular. The tumour had a considerable range of mobility from side to side, but less in the vertical direction. The circumference of the abdomen at the umbilicus was $36 \frac{1}{2}$ inches. On vaginal examination a lump was felt bimanually to the left which was taken for the body of the uterus, and the sound passed into this lump a distance of three inches. I was not sure whether the tumour was a large subperitoneal fibroid or an ovarian tumour.

Operation was performed on August 3rd, 1899. On opening the abdomen the tumour was found to be a large subperitoneal fibroid of the uterus attached to the uterus by a pedicle as thick as the wrist. A loop of intestine some eight inches long was festooned across the front of the tumour and was adherent to it by broad membranous adhesions containing large vessels. In commencing to separate and tie these the surface of the tumour from which 
the adhesions were detached bled so freely that it was necessary to put an elastic ligature tightly round the pedicle of the tumour. This controlled the bleeding. After separating all the adhesions peritoneal flaps were made from about two inches above the elastic ligature and the tumour (which weighed five pounds and six ounces) was then cut away. On loosening the elastic ligature the cut surface bled freely, but this was stopped by successive silk sutures passed rather deeply and tied as tightly as possible. The flaps of peritoneum were then sewn over the \& tump. The left Fallopian tube formed a hydrosalpinx and it was accordingly removed. The right uterine appendages were also removed with a view to check the development of other small fibroids. The body of the uterus in this case was not removed. A Keith's drainage-tube was inserted and the rest of the abdomen was closed in the usual way. The patient did perfectly well. Some months after leaving the hospital she came up again to tell us that she continued quite well.

CASE 9. Uterus enlarged by fibroids furming a tumour rising to tro inches above the navel ; portion of the tumour firmly fixed by adhesions in Douglas's pouch bulging down the posterior vaginal wall; abdominal hysterectomy with intra-peritoneal treatment of the stump; good recovery. - A single woman, aged 29 years, was admitted into the London Hospital on August 8th, 1899. The patient was sent to me by my colleague, Dr. G. Schorstein. The patient was a Russian Jewess and could speak very little English, so that the history was obtained with some difficulty through an interpreter. She seemed to have had pain in the lower abdomen more or less for three years. The periods had lasted eight days and during the first three days she had lost a great deal of blood. Her pain was much worse during the period. There had been no intermenstrual loss. believe the obvious enlargement of the abdomen was one cause of considerable concern to the patient.

On examination a firm, rounded, irregular tumour was felt occupying the lower part of the abdomen and reaching to three fingers' breadth above the umbilicus; it extended outwards some four inches from the middle line on each side. The tumour was fairly moveable from side to side but not vertically. Vaginal examination had to be made under anæsthesia as the hymen was perfect and did not admit the finger without tearing. The os uteri was very high up, about half an inch above the pubes and to the right; it could only be felt by pressing down the tumour. A hard convex portion of the tumour lay in the posterior fornix bulging down the posterior vaginal wall to within one and a half inches of the vaginal orifice. This mass, which evidently formed part of the abdominal tumour, was practically fixed. The sound could only be passed tisree inches.

Operation was performed on August 18th, 1899. A large incision was made, which extended some three inches above the umbilicus. A large tumour was found in the fundus and posterior wall of the uterus extending well out to the sides, so as to leave very little room for manipulation. There was a second tumour almost distinct from the first growing from the posterior wall of the uterus and lying in the pelvis to which it was firmly adherent. This was the mass that had been felt lying in the posterior fornix on vaginal examination. There was also on the right side an ovarian tumour of the size of a goose's egg. On the left side the ovary was larger than normal and slightly cystic. With great difficulty the upper part of the broad ligament was secured and divided on each side; a peritoneal flap was reflected from the anterior wall of the uterus and the uterine arteries were tied. Owing to the tumour described as growing from the lower part of the posterior wall of the uterus being firmly adherent in the pelvis no posterior flap of peritonenm could be made in this case. The tumour in question was freed from its adhesions, leaving a rather extensive raw surface in the neighbourhood of Douglas's pouch. It was not till after this step of the operation that the uterus could be drawn out of the wound. The uterus and fibroid tumour were removed at the level of the internal os. Both uterine appendages were removed with the tumour. The stump was covered as well as possible with the anterior flap of peritoneum, which had been cut long. A Keith's tube was inserted and the rest of the wound, which measured seven inches in length, was closed as usual. The patient made a very good recovery and left the hospital on Sept. 19th. I have seen her once some months afterwards and she was then quite well.

Harley-street, $w$

\section{ON THE RESTORATION OF COORDINATED MOVEMENTS AFTER NERVE- SECTION. 1}

\author{
By ROBERT KENNEDY, M.A., D.Sc., M.D. GlasG.
}

IT is now well known that after reunion of a divided nerve by means of suture function may return sooner or later, and that not only does this restoration of function imply simple return of sensation and of the capacity of making voluntary movements, but the re-establishment of complete localisation of sensation and of coördination of movements. When it is remembered that the individual nerve-fibres are supposed to be paths between well-defined centres and peripheral endings it is clear that so perfect a restoration of function is in many respects remarkable. Thus in coapting a divided nerve it is not likely that this can be effected with so great accuracy that the corresponding ends of the divided nerve-fibres can be brought into apposition. It is more likely that in coapting the nerve most of the ends of the nerve-fibres which are brought into contact are ends wbich do not correspond; and thus it would be expected that in the reunited nerve new paths for the nervous impulses would be established. And yet, despite this complexity of structure and of function, the reunited nerve seems to be as capable as before the division of subserving its functions. In suturing divided nerves, care is usually given to secure that the two segments are approximated as nearly as possible in their old relationship. Thus the perfect recovery of localised and coördinated function might be due to a majority of the fibres being thus placed in a position for union of corresponding ends to take place.

To investigate this subject, three experiments were per. formed on dogs, the sciatic nerve being chosen. In two experiments the peripheral segment, before reunion by means of suture, was twisted so as to bring the maximum number of non-corresponding nerve-fibre ends into juxtaposition, while in the third experiment reunion was made as accurately in the old position as possible. The object of this was to ascertain (1) if the time taken for the first evidence of recovery of coördinated movements and the course of development of the same were identical or different in the two cases; and (2) if the resulting cicatricial nerve segments showed microscopically any important differences in the arrangement of the nerve-fibres. In all three experiments, the animal being anæsthetised, the sciatio nerve was divided at the level of the trochanter; but in the first two, before coapting the two ends by suture, the peripheral segment was rotated to the extent of a semicircle. Thus, on coaptation, the fibres of one side of the central segment were brought into apposition with fibres with which formerly they did not connect-i.e., the fibres of the opposite side of the nerve. In the third experiment the nerve was divided at the same point and accurate coaptation in the old relationship was effected by suture. The following is the course which the experiments exhibited with reference to recovery of function.

EXPERIMENT 1. Division of the sciatic nerve at the level of the trochanter; rotation of the peripheral segment to the extent of a semicircle and suture.-Until the seventh day after the operation the animal showed no sign of returning function. It walked on the limb which was supported by plaster-of. Paris bandages. The paw was left unsupported for examination purposes and until the seventh day while the animal walked on the limb the paw was dragged along the ground with the dorsal surface down. But on the seventh day the paw was first used normally in walking, the plantar surface being placed correctly on the ground. This was taken as evidence of returning coördinated movements. Frequently while walking the paw turned over and the animal rested on the dorsal surface, but on these occasions the normal position was voluntarily regained after a step or two. Restoration of localised sensation could not be ascertained, but undoubted evidence of sensation was obtained on the tenth day. On the fourteenth day the supporting plasterof-Paris splint was $r \in$ moved and the wc und was found to be

1 The research is published in extenso in the Transastions of the

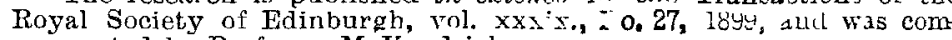
muncated by Professor McKendric'z. 